\title{
Overview of Cancer Survivorship Care for Primary Care Providers
}

\author{
Sukesh Manthri ${ }^{1}$, Stephen A. Geraci ${ }^{2}$, Kanishka Chakraborty ${ }^{3}$ \\ 1. Oncology, East Tennessee State University, Johnson City, USA 2. Internal Medicine and Medical Education, East \\ Tennessee State University, Johnson City, USA 3. Medical Oncology, East Tennessee State University, Johnson City, \\ USA
}

Corresponding author: Sukesh Manthri, sukeshmanthri123@gmail.com

\begin{abstract}
Survivorship care for a patient with cancer is often complex and requires a multidisciplinary approach. Cancer and its treatment can have late and long-term physical and psychosocial effects. After the acute and intense period of treatment and surveillance administered by oncology teams, cancer survivors slowly transition care to primary providers. Cancer survivors then enter into an extended phase of survivorship whether they are cancer-free, in remission, or living with cancer. In this phase, symptoms related to cancer and its treatment may vary over time. Developing a care plan can facilitate the transition of care between all providers taking care of cancer patients.
\end{abstract}

Categories: Family/General Practice, Internal Medicine, Oncology

Keywords: cancer, survivorship, primary care

\section{Introduction And Background}

The American Cancer Society (ACS) estimates 1.8 million new cases of cancer will be diagnosed in 2020. A third of these patients will die from their malignancy [1]. According to statistics completed in January 2019, about 16.9 million people are currently alive in the United States after being diagnosed with cancer (this represents $5.0 \%$ of the population), a number that is expected to rise by $\geqslant 5$ million by the year 2024 [2]. The cost of cancer care in the U.S. is rising and is expected to reach around $\$ 173$ billion by 2020 [3].

The National Cancer Institute (NCI) definition is the most widely accepted: "An individual is considered a cancer survivor from the time of diagnosis, through the balance of his or her life." Family members, friends, and caregivers are also impacted by the survivorship experience and are therefore included in this definition [4]. Cancer survivors face multiple effects of both their cancer and its treatment, most of which can develop during treatment or manifest years after active therapy is concluded [5]. At least $50 \%$ of cancer survivors will have some consequences affecting their lives due to cancer and its' treatment [6]. Major/frequent concerns for survivorship assessment includes cardiac toxicities, anxiety and depression, fatigue, pain syndromes, sexual dysfunction, sleep disorders, and the ability to maintain a healthy lifestyle. Many organizations have developed standards for the care of cancer survivors including the ACS, George Washington University,

Received 05/24/2020

Review began 06/06/2020 Review ended 08/25/2020 Published 09/02/2020

(c) Copyright 2020 Manthri et al. This is an open access article distributed under the terms of the Creative Commons Attribution License CC-BY 4.0., which permits unrestricted use, distribution, and reproduction in any medium, provided the original author and source are credited.
Key components of survivorship care plan include proper surveillance of cancer spread and recurrence, ongoing evaluation of the effects of cancer and its treatment on a patient, proper management of such effects, and coordination of care among all providers taking care of the cancer survivor [9]. The role of the primary care provider (PCP) in managing survivorship care is becoming even more important as the population of cancer survivors grows [10]. A 2009 survey conducted by the NCI and the ACS found that $>50 \%$ of PCPs report that they administer survivorship care. PCPs with training on the effects of cancer and its treatment, who employ good communication in a co-management model with oncologists, were more likely to provide survivorship care [11]. However, the role of the PCP in survivorship care is not formally described [12]. This review will explore the common effects of cancer and its treatment including psychosocial effects, detection of secondary cancers, major long-term toxicities, and late side effects.

\section{Review}

\section{Psychosocial effects}

Anxiety, depression, and post-traumatic stress disorders are the most common psychosocial consequences of cancer and its treatment. Together they affect $25-30 \%$ of cancer survivors [13]. Moreover, cancer survivors may develop altered social relationships, feelings of discrimination, and work-related difficulties. These may be related to fear of recurrence and changes in body image [14]. Assessment for psychosocial complications should be performed at least annually for all cancer survivors [15]. More detailed assessments should be given to patients at higher risk of psychosocial consequences (e.g., prior history of psychiatric disease, low social-economic status) [16]. Addressing these issues requires an inter-disciplinary approach that includes primary care providers, oncologists, psychiatrists, and others. This approach should employ 
psychopharmacological and psychological interventions to reduce psychosocial stressors and treat psychiatric illness on the cancer survivor and his/her caretakers [17].

\section{Detection of second primary cancers and cancer recurrence}

Surveillance for cancer recurrence, an integral part of survivorship care, is usually carried out for five years for most types of cancer. Depending on the type of primary malignancy, a combination of history-taking and physical examination coupled with laboratory and surveillance imaging are employed. Assessment and testing typically conform to a guideline-driven timeline (e.g., from the National Comprehensive Cancer Network [NCCN]). It is usually carried out by the oncologists and their teams. On the other hand, the detection of second primary cancers is usually carried out by the PCP. Second primary cancers in survivors are detected at a higher rate (2-30\%) depending upon initial primary cancer type, compared to the general population [6]. Explanations for this observation include predisposing genetic disease, prior exposure to chemotherapy and/or radiotherapy, and the shared causative factor (i.e., a risk factor for both types of cancer) [18]. Most recently, the ACS recommended screening for secondary malignancies similar to screening for initial cancers in the general population. PCPs should carry a discussion about the risks and benefits of a particular screening modality with each patient. Some survivors may require screening for secondary malignancy according to recommendations that differ from the general population. One example is female carriers of Lynch syndrome who require cancer screening at the age of 35 (lifetime increased risk for cancers - colorectal, endometrial, urinary tract, pancreatic or biliary, ovary, gastric, small intestine and others). Another example is female survivors who received thoracic radiation as part of Hodgkin's lymphoma treatment will require breast cancer screening with MRI. Communication between treating oncologists and primary providers is required in such patients [19].

\section{Long-term toxicities and late side-effects}

Cancer survivors are at high risk to develop complications secondary to cancer and its treatments. The ACS, American Society of Clinical Oncology (ASCO), and the NCCN periodically publish survivorship care guidelines. Table 1 summarizes common complaints of cancer survivors and the recommended approach for addressing them. Moreover, some cancer patients receive long-term (years) treatment with a cytotoxic agent, hormonal treatment, androgen deprivation therapy, targeted therapy, or immunotherapy. Primary care physicians should be aware of the common toxicities of the most commonly-used cancer therapies (Table 2).

\begin{tabular}{|c|c|c|c|}
\hline Problem & $\begin{array}{l}\text { Common } \\
\text { Symptom }\end{array}$ & Related Malignancy & Recommendation \\
\hline \multirow[t]{3}{*}{ GI Issues } & Obstruction & Colon, rectal, and anal malignancies & $\begin{array}{l}\text { Discuss bowel habits, rectal bleeding, and sphincter } \\
\text { dysfunction }\end{array}$ \\
\hline & $\begin{array}{l}\text { Rectal } \\
\text { bleeding/ulcers }\end{array}$ & & Evaluate for incisional hernia \\
\hline & $\begin{array}{l}\text { Challenges with } \\
\text { ostomy care }\end{array}$ & & Refer to an appropriate specialist \\
\hline \multirow[t]{2}{*}{ Dental/Oral } & Loss of taste & Head and neck & Adequate oral hygiene \\
\hline & $\begin{array}{l}\text { Xerostomia } \\
\text { hyposalivation }\end{array}$ & & Evaluate for nutritional deficiencies \\
\hline & Dental caries & & Referral to a specialist (ENT, dentists) \\
\hline \multirow[t]{3}{*}{$\begin{array}{l}\text { Fatigue (rating in a } \\
\text { scale of } 0-10 \text { ) }\end{array}$} & Tiredness & Common to most & Use of an assessment tool (MDASI, BFI) \\
\hline & & & Optimize nutrition and physical activity \\
\hline & & & $\begin{array}{l}\text { Assess for causative etiology: anemia, thyroid dysfunction, } \\
\text { sleep disorder }\end{array}$ \\
\hline \multirow[t]{3}{*}{ Pain } & Somatic & Common to most & $\begin{array}{l}\text { Assess using pain scale and comprehensive history and } \\
\text { exam }\end{array}$ \\
\hline & Neuropathic & & $\begin{array}{l}\text { Offer intervention (NSAID, antidepressants, } \\
\text { anticonvulsants, opioids, physical activity) }\end{array}$ \\
\hline & & & Refer to a specialist once etiology is determined \\
\hline Lymphedema & & Breast & Offer compressive garment \\
\hline
\end{tabular}




\section{Cureus}

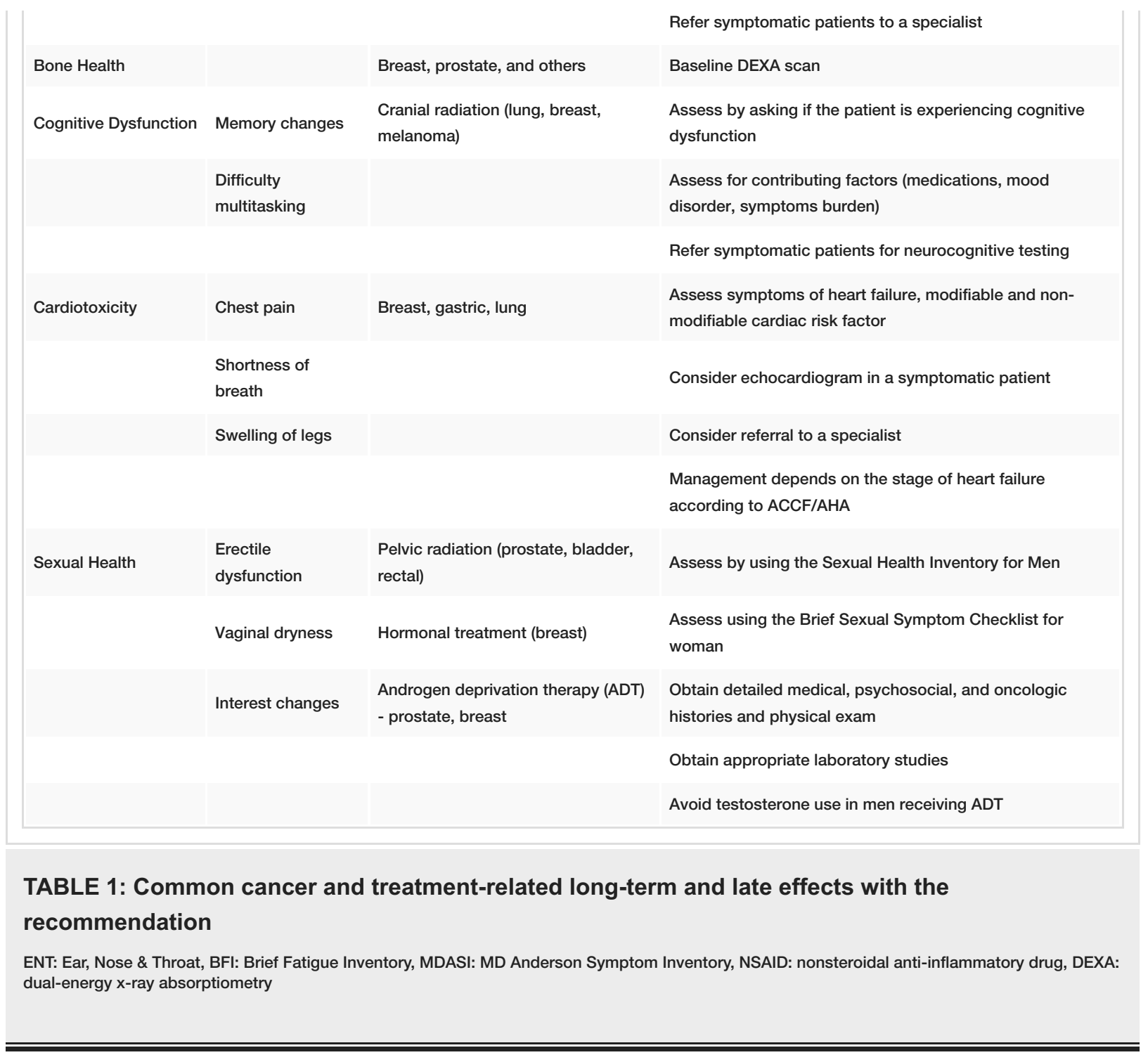




\section{Cureus}

\begin{tabular}{|c|c|c|}
\hline Therapy & Category & Serious side effect \\
\hline \multicolumn{3}{|l|}{ Cytotoxic } \\
\hline Anthracycline (doxorubicin) & Antibiotic & Cardiotoxicity \\
\hline Taxane (paclitaxel, docetaxel) & Mitotic inhibitors & Peripheral neuropathy \\
\hline Platins (cisplatin, carboplatin) & Alkylating agent & Peripheral neuropathy, ototoxicity \\
\hline Bleomycin & Inhibition of DNA synthesis & Pneumonitis, pulmonary fibrosis \\
\hline Capecitabine & Antimetabolite & Hand-foot syndrome \\
\hline Vincristine & Mitotic inhibitors & Peripheral neuropathy \\
\hline Cyclophosphamide & Alkylating agent & Secondary malignancy \\
\hline Etoposide & Topoisomerase II inhibitor & Secondary malignancy \\
\hline \multicolumn{3}{|l|}{ Monoclonal antibodies } \\
\hline Bortezomib & Proteasome inhibitor & Peripheral neuropathy \\
\hline Bevacizumab & VEGF inhibitor & Hypertension, thromboembolic events \\
\hline Trastuzumab & HER-2 inhibitor & Cardiotoxicity \\
\hline \multicolumn{3}{|l|}{ Targeted therapy } \\
\hline Everolimus & mTOR inhibitor & Pneumonitis, hyperglycemia and, dyslipidemia \\
\hline Nilotinib & Tyrosine kinase inhibitor (TKI) & QT prolongation \\
\hline Vemurafenib & B-Raf enzyme inhibitor & Squamous cell carcinoma \\
\hline Sorafenib & TKI & Hemorrhage, hepatoxicity \\
\hline Ibrutinib & Bruton's tyrosine kinase (BTK) inhibitor & Peripheral edema, atrial fibrillation, bruising \\
\hline Idelalisib & PIK3 inhibitor & Colitis, hepatitis, pneumonitis \\
\hline \multicolumn{3}{|l|}{ Endocrine treatment } \\
\hline Tamoxifen & Selective ER modulator & Thromboembolism, endometrial cancer \\
\hline Aromatase Inhibitors & & Osteoporosis, vaginal dryness, arthralgia \\
\hline Androgen-deprivation therapy & & sexual and cardiovascular dysfunctions Osteoporosis \\
\hline \multicolumn{3}{|l|}{ Immunotherapy } \\
\hline Ipilimumab & anti-CTLA & Colitis \\
\hline Nivolumab & ati-P & jidism \\
\hline
\end{tabular}

\section{TABLE 2: Common toxicities of the most commonly-used cancer therapies}

VEGF: vascular endothelial growth factor, HER-2: human epidermal growth factor receptor 2, mTOR: mammalian target of rapamycin, PIK3: phosphoinositide 3-kinase, CTLA: cytotoxic T-lymphocyte-associated protein, PD-1: programmed death-1

\section{The financial burden of cancer care}

Based on NCI cancer trends progress reports, national expenditures associated with cancer have been steadily increasing in the United States. Cancer is one of the most expensive medical conditions to treat in the United States. Medical care expenditures for the care of cancer survivors accounted for an estimated $\$ 137.4$ billion in the United States in 2010 and are expected to rise to as high as $\$ 173$ billion by 2020 . National expenditures were largest for female breast, colorectal, prostate, lymphoma, and lung cancers, reflecting the prevalence of the disease, treatment patterns, and costs of care. Estimates of national expenditure in 2018 for breast cancer care is $\$ 19,700$ million, colorectal is $\$ 16,630.9$ million, prostate is $\$ 15,299.2$ million, lymphoma is $\$ 14,626.7$ million, and lung cancer is $\$ 14,185.5$ million. As the population ages, cancer prevalence will increase even if cancer incidence rates remain constant or decrease somewhat. 
Costs are also likely to increase as new, more advanced, and more expensive treatments are adopted as standards of care. Financial toxicity in cancer survivors may lead to not taking medications as directed, having a lower quality of life, and debt and bankruptcy. Cancer survivors may have financial problems many years after they are diagnosed due to ongoing payment for ongoing cancer treatment or care for late effects from their treatment [20].

\section{Special considerations like the impact of pandemics like COVID-19 on cancer survivors}

The coronavirus disease 2019 (COVID-19) pandemic has raised challenges and increased the complexity of cancer care. Important questions include balancing the risk from treatment delay versus harm from COVID19, limiting the use of immunosuppressive cancer treatments whenever possible, mitigating appropriately and fairly allocating limited healthcare resources. Limited healthcare resources can lead to delays in care delivery, screening, and surveillance. Patients with long-term immune suppression may be at increased risk of infection. Evolving data indicate a high rate of severe disease and mortality from COVID-19 in patients with lung cancer. The impact on cancer patients cannot be quantified at this moment and will be determined in upcoming months.

\section{Specific tumor types}

Testicular Cancer

Testicular cancer (TC) survivors present a challenging population. The incidence of TC in the U.S. has increased in the past 20 years in men aged 18 to 39 years [21]. Chemotherapy is the mainstay of treatment. A combination of cisplatin, bleomycin, vinblastine, and ifosfamide is commonly used. The relative 10-year survival rates approach 95\% for all patients with TC [22]. Survivors have a 1.5-4.0-fold increased risk for developing a second malignancy. The risk is even higher (a 5.9-fold increase) in patients who had received radiotherapy and chemotherapy [9]. Although there is no consensus on surveillance in this group, many experts recommend that TC survivors undergo cancer screenings, which is applicable to the general population [18]. Moreover, TC survivors are at an increased risk for cardiovascular disease and pulmonary toxicity. Approximately $7-21 \%$ of TC patients who receive bleomycin will develop pulmonary toxicity. If TC survivors require surgery any time after treatment, a thorough cardiopulmonary evaluation is recommended $[23,24]$. Finally, hypogonadisms and infertility are common among TC survivors. Aging, chemotherapy, orchiectomy, and radiotherapy all contribute to infertility. Young men who have not completed their family are required to be counseled for sperm preservation. This adds to the cost of the treatment as the patient needs to pay for the preservation and maintenance of the sample. Low testosterone levels and increased serum concentrations of luteinizing hormone and follicle-stimulating hormone are commonly detected. The risk for testosterone deficiency is 3.3- to 5.2-fold higher in TC survivors than in the general population. Testosterone replacement is often attempted, but no data support this approach [25]. All cancer survivors should undergo continuous efforts to promote a healthy lifestyle, including consuming a balanced diet and smoking abstinence.

\section{Head and Neck Cancer}

The structure and function of the head and neck allow for many critical processes including swallowing, speaking, airway patency maintenance, and taste. Head and neck cancer (HNC) patients typically require intensive multi-disciplinary care from otolaryngologists, medical and radiation oncologists, and other healthcare providers (dentists, nutritionists, gastroenterologists, etc). Early-stage and node-negative HNC can be treated with surgery or radiotherapy alone, while locally advanced HNC require chemotherapy (platinum-based) as part of the treatment plan. Functional loss, vascular complications, and hypothyroidism are frequently discovered in HNC survivors. The incidence of esophageal strictures leading to dysphagia is $20-30 \%$, with $82 \%$ of these presenting within the first post-treatment year [26]. Discordance often exists between the clinicians and the patient's judgment of dysphagia. Aspiration presenting with chronic cough, frequent pneumonia, and weight loss is another frequent complaint by HNC survivors. However, up to 50\% of chronic aspirators can be silent and are only detected by swallowing studies [27]. There is a role for cancer rehabilitation, including speech and language therapists with a therapy consisting of swallowing exercises and maneuvers, neuromuscular stimulation, and education regarding the importance of lifestyle modification (eg, changing the food consistency to prevent aspiration) can help prevent worsening (or more significant) morbidity. Carotid artery compromise may also occur, though routine ultrasound screening is not supported by data [28]. Dental caries secondary to xerostomia is also common (24\%), and nutritional status and oral health should be assessed routinely [29]. Alcohol-free rinses can be used to rehydrate the oral mucosa and prevent crusting. Avoidance of caffeine, spicy foods, and tobacco should be encouraged in HNC survivors [27]. Conversely, hypothyroidism is a late post-treatment complication; thyroid stimulating hormone (TSH) should be checked at six- to 12-month intervals as recommended by NCCN. Finally, jaw pain or swelling may signal osteonecrosis. Early conservative management with daily chlorhexidine gluconate and broad-spectrum antibiotics coupled with early referral to oral surgeons can prevent osteonecrosis of jaw from progressing [30].

Lung Cancer 
Lung cancer, the most common cause of cancer death in the U.S., is common, with the majority of patients diagnosed after age 60 years. ACS 2013 estimated 412,230 lung cancer survivors. The IOM recommends developing care plans for cancer survivors. This is particularly more valuable to lung cancer survivors giving the complexity of lung cancer treatments. The care plan, prepared by the oncology team, should include a summary of treatments provided including possible side effects and formal follow up plans [31]. Care plans would assist in the sharing of knowledge and patients care among oncologists and primary providers. Lung cancer patients are most often (though not always) long-term smokers and are exposed to various treatment modalities (medical therapy, radiation, and surgery), placing survivors at particularly high risk for developing secondary malignancies (largely owing prior exposure to causative factors and exposure to therapy). Risk of lung, larynx, and bladder secondary malignancies remains elevated even 10 years after initial diagnosis [32]. Respiratory complaints (cough, dyspnea, and reduced exercise tolerance) are among the most common complaints among lung cancer survivors. Survivorship care should focus on counseling on smoking cessation, optimizing treatment of chronic obstructive lung disease (COPD, a frequent co-morbid condition), and promoting an overall healthy lifestyle [33]. Physicians should realize it is never too late to council lung cancer patients on smoking cessation. Uncontrolled cancer-related pain is reported in $45 \%$ of cancer patients [34]. Pain is commonly associated with anxiety and depression leading to disability and impaired quality of life (QOL) among lung cancer survivors. Post thoracotomy pain syndrome affects $80 \%$ of lung cancer patients receiving surgical resection. Thirty percent of lung cancer survivors had chronic, mild post-thoracotomy pain lasting four years post-resection. Other factors impacting QOL in cancer survivors are distress and fear of recurrence. Distress is reported in 25-40\% of cancer survivors while fear of recurrence ranges from 5-89\%. Both factors are more pronounced in lung cancer survivors. Distress and fear of recurrence vary over time in cancer survivors and may be triggered by the onset of unexplained symptoms. Social support and coping strategies can have a positive impact on QOL [35].

\section{Colon Cancer}

The overall five-year survival rate for colorectal cancers (CRC) has increased to $~ 65 \%$ in recent years. Moreover, the survival of stage IV CRC has also improved with the majority of patients receiving maintenance therapy for an extended period of time [36]. Bowel dysfunction is common among CRC survivors. Up to $50 \%$ of patients may report diarrhea lasting more than four weeks and affecting lifestyle, requiring anti-diarrheal agents. Chemotherapy-induced peripheral neuropathy (C-IPN) is also common (40\%), especially in patients receiving oxaliplatin ( $>900 \mathrm{mg} / \mathrm{m} 2$ cumulative dose). Only about half of these patients will experience a total resolution of symptoms [36]. Patients with diabetes and prior alcohol abuse are particularly susceptible. Unfortunately, no data support any preventive measures. Duloxetine, $30 \mathrm{mg}$ daily for one week followed by $60 \mathrm{mg}$ daily for four weeks, appears to be beneficial in reducing neuropathic symptoms [37]. Urogenital and sexual dysfunction is seldom acknowledged as a long-term complication, especially in women receiving radiotherapy for rectal cancer [38]. Dyspareunia may be reduced with vaginal lubricants. Male CRC survivors with erectile dysfunction may benefit from oral phosphodiesterase- 5 inhibitors [39]. Finally, bladder dysfunction should be assessed to determine whether symptoms are from a hypo-contractile vs hypoactive bladder, and treatment should be based on such assessment.

\section{Prostate Cancer}

Prostate cancer (PC) survivors share common issues with breast cancer survivors (see below). The majority of men diagnosed with PC are 60-70 years old. More than $90 \%$ of afflicted patients present with the local or regional disease for which the expected five-year survival approaches 100\% [40]. Men with local or regional PC who had received radiotherapy and are receiving only androgen deprivation therapy (ADT) are an important subset of PC patients. ADT increases the risks of cardiovascular disease, osteoporosis, and sexual dysfunction. Clinicians should follow the same screening and prevention protocols for cardiovascular disease as recommended for the general population. Men on ADT should have a DEXA scan (dual-energy xray absorptiometry) and FRAX (world health organization fracture risk assessment tool) score calculated. Bisphosphonate and denosumab are FDA-approved for PC patients on ADT who qualify for osteoporosis treatment. Sexual dysfunction can be assessed by screening tools such as the Sexual Health Inventory for Men [41]. A trial of phosphodiesterase type 5 inhibitors, urologic consultation, and/or psychotherapy evaluation are potential interventions. After definitive radical prostatectomy is completed, prostate-specific antigen (PSA) serum concentrations should be undetectable by two months after surgery [42]. After definitive radiation therapy (RT) with or without ADT is completed, PSA increase by $2 \mathrm{ng} / \mathrm{mL}$ or more above the nadir PSA is the standard definition for PSA persistence/recurrence and a recurrence evaluation should be considered. The same conception is applied when PSA has been confirmed to be increasing after RT even if the increase above nadir is not yet $2 \mathrm{ng} / \mathrm{mL}$, especially in candidates for salvage local therapy who are young and healthy [43]. NCCN recommends measuring serum PSA every six to 12 months for the first five years after treatment, then annually and an annual digital rectal exam. Any recurrence or persistence in PSA or new mass on palpation should prompt referral to primary treating specialist for consideration for surgery or radiation based on initial definitive therapy [44].

\section{Breast Cancer}

Almost four of every 10 female cancer survivors had breast cancer (BC) as their primary malignancy, with an estimated 3.6 million breast cancer survivors in the U.S. as of 2016-2017. Breast cancer treatment has 
improved significantly in the past decade, reflected in a five-year survival rate of almost $90 \%$ among all breast cancer patients [45]. The treatment approach for BC varies with the patient's clinical-stage, menopausal status, and tumor-specific characteristics (hormone receptors, HER-2 neu status). Treatment may include surgical resection (lumpectomy or mastectomy), radiotherapy, chemotherapy, and/or hormonal treatment. Some patients may require adjuvant endocrine therapy (aromatase inhibitor by suppressing plasma estrogen levels or selective estrogen receptor modulator by competitive antagonism of the estrogen receptor) for the woman with hormonal receptor-positive $\mathrm{BC}$ for a period of five to 10 years. $\mathrm{BC}$ survivors are at a high risk of cardiac toxicity. Prior exposure to radiation, anthracyclines, trastuzumab, and aromatase inhibitors can all contribute to progressive cardiac dysfunction [46]. Clinicians should assess symptoms of cardiac disease, monitor lipid levels, and prescribe preventive measures including therapeutic lifestyle changes. Also, exposure to these therapies, combined with increasing age, lack of exercise, vitamin D deficiency, and low serum calcium levels, all increase the likelihood of osteoporosis in BC survivors. A repeat DEXA scan testing is advised one or two years after starting treatment for a woman on an aromatase inhibitor or who experiences ovarian failure secondary to chemotherapy when the results are likely to influence clinical management. Calcium (1200 mg daily) and vitamin D (600 IU daily) should be prescribed while BC survivors are receiving an aromatase inhibitor per ACS guidelines [47]. The PCP should assess for body image concerns which affect $31-67 \%$ of BC survivors, and an even higher incidence is seen among younger women. Clinicians should assist patients in obtaining prosthetic devices, wigs, and other resources to improve their body image. Surgical interventions (reconstruction/prosthetic implantation) and mental health referral may be helpful in select cases.

\section{Conclusions}

Caring for cancer survivors is a collaborative effort. Most cancer patients will have a close follow-up with their primary specialist during the intense period of cancer treatment. Survivors may slowly transition care from their subspecialist to their PCP. The IOM recommends that treating specialists provide survivorship care plans, which include a treatment summary and follow-up recommendations, to the PCP when the transition of care begins. PCPs should understand the most common long-term and late effects of common cancers and their treatments. They should also have access to common survivorship resources.

\section{Additional Information \\ Disclosures}

Conflicts of interest: In compliance with the ICMJE uniform disclosure form, all authors declare the following: Payment/services info: All authors have declared that no financial support was received from any organization for the submitted work. Financial relationships: All authors have declared that they have no financial relationships at present or within the previous three years with any organizations that might have an interest in the submitted work. Other relationships: All authors have declared that there are no other relationships or activities that could appear to have influenced the submitted work.

\section{References}

1. Cancer Facts \& Figures 2020. (2020). https://www.cancer.org/content/dam/cancer-org/research/cancerfacts-and-statistics/annual-cancer-facts-and-figures/20...

2. Statistics, Graphs and Definitions. (2020). https://cancercontrol.cancer.gov/ocs/statistics/index.html.

3. Mariotto AB, Yabroff KR, Shao Y, Feuer EJ, Brown ML: Projections of the cost of cancer care in the United States: 2010-2020. J Natl Cancer Inst. 2011, 103:117-128. 10.1093/jnci/djq495

4. About cancer survivorship research: survivorship definition . (2014). https://cancercontrol.cancer.gov/ocs/

5. Stein KD, Syrjala KLc, Andrykowski MA: Physical and psychological long-term and late effects of cancer . Cancer. 2008, 112:2577-2592. 10.1002/cncr.23448

6. Valdivieso M, Kujawa AM, Jones, T, Baker LH: Cancer survivors in the United States: a review of the literature and a call to action. Int J Med Sci. 2012, 9:163-173. 10.7150/ijms.3827

7. American Cancer Society Survivorship Care Guidelines . (2020). https://www.cancer.org/health-careprofessionals/american-cancer-society-survivorship-guidelines.html.

8. The Cancer Survivorship E-Learning Series for Primary Care Providers Communication Toolkit . https://smhs.gwu.edu/cancercontroltap/sites/cancercontroltap/files/ESeries\%20Communication\%20Toolkit\%20FINAL\%20Co-br....

9. Travis LB, Fossa SD, Schonfeld SJ, et al.: Second cancers among 40,576 testicular cancer patients: focus on long-term survivors. J Natl Cancer Inst. 2005, 97:1354-65. 10.1093/jnci/dji278

10. Nekhlyudov L, Aziz, NM, Lerro C, Virgo KS: Oncologists' and primary care physicians' awareness of late and long-term effects of chemotherapy: implications for care of the growing population of survivors. J Oncol Pract. 2014, 10:29-36. 10.1200/JOP.2013.001121

11. Klabunde CN, Han PK, Earle CC, et al.: Physician roles in the cancer-related follow-up care of cancer survivors. Fam Med. 2013, 45:463-74.

12. Khatcheressian JL, Wolff AC, Smith TJ: American Society of Clinical Oncology 2006 update of the breast cancer follow-up and management guidelines in the adjuvant setting. J Clin Oncol. 2006, 24:5091-7. 10.1200/JCO.2006.08.8575

13. Mitchell AJ, Chan M, Bhatti H: Prevalence of depression, anxiety, and adjustment disorder in oncological, haematological, and palliative-care settings: a meta-analysis of 94 interview-based studies. Lancet Oncol. 2011, 12:174. 10.1016/S1470-2045(11)70002-X

14. Crespi CM, Ganz PA, Petersen L, Castillo A, Caan B: Refinement and psychometric evaluation of the impact 
of cancer scale. J Natl Cancer Inst. 2008, 100:1530-1541. 10.1093/jnci/djn340

15. NCCN Guidelines on Survivorship. (2019).

https://www.nccn.org/professionals/physician_gls/pdf/survivorship.pdf.

16. Andersen BL, DeRubeis RJ, Berman BS: Screening, assessment, and care of anxiety and depressive symptoms in adults with cancer: an American Society of Clinical Oncology Guideline Adaptation. J Clin Oncol. 2014, 32:1605-1619. 10.1200/JCO.2013.52.4611

17. Rubin G, Berendsen A, Crawford SM, Dommett R, Earle C, Emery J, Zimmermann C: The expanding role of primary care in cancer control. Lancet Oncol. 2015, 16:1231-1272. 10.1016/S1470-2045(15)00205-3

18. Wood ME, Vogel V, Ng A, et al.: Second malignant neoplasms: assessment and strategies for risk reduction . J Clin Oncol. 2012, 30:3734-3745. 10.1200/JCO.2012.41.8681

19. Smith RA, Manassaram-Baptiste D, Brooks D, et al.: Cancer screening in the United States, 2015: a review of current American Cancer Society guidelines and current issues in cancer screening. CA Cancer J Clin. 2015, 65:30-35. 10.3322/caac.21261

20. Financial Toxicity (Financial Distress) and Cancer Treatment (PDQ $\left.{ }^{\circledR}\right)$-Patient Version . (2019). https://www.cancer.gov/about-cancer/managing-care/track-care-costs/financial-toxicity-pdq.

21. Siegel R, Ma J, Zou Z, et al.: Cancer statistics. CA Cancer J Clin. 2014, 64:9-29. 10.3322/caac.21208

22. Hanna N, Einhorn LH: Testicular cancer: a reflection on 50 years of discovery . J Clin Oncol. 2014, 32:308592. 10.1200/JCO.2014.56.0896

23. Fossa SD, Gilbert E, Dores GM, et al.: Noncancer causes of death in survivors of testicular cancer . J Natl Cancer Inst. 2007, 99:533-44. 10.1093/jnci/djk111

24. Donat SM: Peri-operative care in patients treated for testicular cancer. Semin Surg Oncol. 1999, 17:282-8. 10.1002/(sici)1098-2388(199912)17:4<282::aid-ssu10>3.0.c0;2-v

25. Fung C, Fossa SD, Williams A, Travis LB: Long-term morbidity of testicular cancer treatment. Urol Clin North Am. 2015, 42:393-408. 10.1016/j.ucl.2015.05.002

26. Laurell G, Kraepelien T, Mavroidis P, et al.: Stricture of the proximal esophagus in head and neck carcinoma patients after radiotherapy. Cancer. 2003, 97:1693-1700. 10.1002/cncr.11236

27. Cohen EE, LaMonte SJ, Erb NL: American Cancer Society Head and Neck Cancer Survivorship Care Guideline. CA Cancer J Clin. 2016, 66:203-239. 10.3322/caac.21343

28. Murphy BA, Deng J: Advances in supportive care for late effects of head and neck cancer . J Clin Oncol. 2015, 33:3314-3321. 10.1200/JCO.2015.61.3836

29. Hong CH, Napeñas JJ, Hodgson BD, et al.: A systematic review of dental disease in patients undergoing cancer therapy. Support Care Cancer. 2010, 18:1007-1021. 10.1007/s00520-010-0873-2

30. Epstein JB, Thariat J, Bensadoun RJ, et al.: Oral complications of cancer and cancer therapy: from cancer treatment to survivorship. CA Cancer J Clin. 2012, 62:400-422. 10.3322/caac.21157

31. Hewitt M, Greenfield S, Stovall E: From Cancer Patient to Cancer Survivor: Lost in Transition . National Academy Press, Washington, DC; 2006.

32. Sarna L, Padilla G, Holmes C, et al.: Quality of life of long-term survivors of non-small-cell lung cancer . J Clin Oncol. 2002, 20:2920-2929. 10.1200/JCO.2002.09.045

33. Choi M, Craft B, Geraci SA: Surveillance and monitoring of adult cancer survivors. Am J Med. 2011, 124:598601. 10.1016/j.amjmed.2010.07.031

34. Rausch SM, Gonzalez BD, Clark MM, et al.: SNPs in PTGS2 and LTA predict pain and quality of life in long term lung cancer survivors. Lung Cancer. 2012, 77:217-223. 10.1016/j.lungcan.2012.02.017

35. Alfano CM, Rowland JH: Recovery issues in cancer survivorship: a new challenge for supportive care . Cancer J. 2006, 12:432-443. 10.1097/00130404-200609000-00012

36. El-Shami K, Oeffinger KC: American Cancer Society Colorectal Cancer Survivorship Care Guidelines. CA Cancer J Clin. 2015, 65:427-455. 10.3322/caac.21286

37. Smith EM, Pang H, Cirrincione C, et al.: Alliance for Clinical Trials in Oncology. Effect of duloxetine on pain, function, and quality of life among patients with chemotherapy-induced painful peripheral neuropathy: a randomized clinical trial. JAMA. 2013, 309:1359-1367. 10.1001/jama.2013.2813

38. Den Oudsten BL, Traa MJ, Thong MS, et al.: Higher prevalence of sexual dysfunction in colon and rectal cancer survivors compared with the normative population: a population-based study. Eur J Cancer. 2012, 48:3161-3170. 10.1016/j.ejca.2012.04.004

39. Park SY, Choi GS, Park JS, Kim HJ, Park JA, Choi JI: Efficacy and safety of udenafil for the treatment of erectile dysfunction after total mesorectal excision of rectal cancer: a randomized, double-blind, placebocontrolled trial. Surgery. 2015, 157:64-71. 10.1016/j.surg.2014.07.007

40. Siegel R, DeSantis C, Virgo K, et al.: Cancer treatment and survivorship statistics, 2012. CA Cancer J Clin. 2012, 62:220-241. 10.3322/caac.21149

41. Cappelleri JC, Rosen RC: The Sexual Health Inventory for Men (SHIM): a 5-year review of research and clinical experience. Int J Impot Res. 2005, 17:307-319. 10.1038/sj.ijir.3901327

42. Stephenson AJ, Kattan MW, Eastham JA, et al.: Defining biochemical recurrence of prostate cancer after radical prostatectomy: a proposal for a standardized definition. J Clin Oncol. 2006, 24:3973-3978. 10.1200/JCO.2005.04.0756

43. Abramowitz MC, Li T, Buyyounouski MK, Ross E, Uzzo RG, Pollack A, Horwitz EM: The Phoenix definition of biochemical failure predicts for overall survival in patients with prostate cancer. Cancer. 2008, 112:55-60. 10.1002/cncr.23139

44. Skolarus TA, Wolf AM, Erb NL, et al.: American Cancer Society prostate cancer survivorship care guidelines . CA Cancer J Clin. 2014, 64:225-49. 10.3322/caac.21234

45. SEER Cancer Statistics Review, 1975-2012. Howlader N, Noone AM, Krapcho M, et al. (ed): National Cancer Institute, Bethesda; 2015.

46. Curigliano G, Cardinale D, Suter T, et al.: Cardiovascular toxicity induced by chemotherapy, targeted agents and radiotherapy: ESMO Clinical Practice Guidelines. Ann Oncol. 2012, 23:155-166.

10.1093/annonc/mds293

47. Runowicz CD, Leach CR, Henry NL, et al.: American Cancer Society/American Society of Clinical Oncology Breast Cancer Survivorship Care Guideline. CA Cancer J Clin. 2016, 66:43-73. 10.3322/caac.21319 